\title{
Intestinal P-glycoprotein exports endocannabinoids to prevent inflammation and maintain homeostasis
}

\author{
Rose L. Szabady, ${ }^{1}$ Christopher Louissaint, ${ }^{1}$ Anneke Lubben, ${ }^{2}$ Bailu Xie, ${ }^{2}$ Shaun Reeksting, ${ }^{2}$ Christine Tuohy, ${ }^{1}$ Zachary Demma, ${ }^{1}$ \\ Sage E. Foley, ${ }^{1}$ Christina S. Faherty, ${ }^{3}$ Alejandro Llanos-Chea, ${ }^{3}$ Andrew J. Olive, ${ }^{1}$ Randall J. Mrsny, ${ }^{2}$ and Beth A. McCormick ${ }^{1}$ \\ 'Department of Microbiology and Physiological Systems, University of Massachusetts Medical School, Worcester, Massachusetts, USA. ${ }^{2}$ Department of Pharmacy and Pharmacology, University of Bath, Bath, \\ United Kingdom. ${ }^{3}$ Mucosal Immunology and Biology Research Center, Division of Pediatric Gastroenterology and Nutrition, Massachusetts Ceneral Hospital and Department of Pediatrics, Harvard Medical \\ School, Boston, Massachusetts, USA.
}

\begin{abstract}
Neutrophil influx into the intestinal lumen is a critical response to infectious agents, but is also associated with severe intestinal damage observed in idiopathic inflammatory bowel disease. The chemoattractant hepoxilin $A_{3}$, an eicosanoid secreted from intestinal epithelial cells by the apically restricted efflux pump multidrug resistance protein 2 (MRP2), mediates this neutrophil influx. Information about a possible counterbalance pathway that could signal the lack of or resolution of an apical inflammatory signal, however, has yet to be described. We now report a system with such hallmarks. Specifically, we identify endocannabinoids as the first known endogenous substrates of the apically restricted multidrug resistance transporter P-glycoprotein (P-gp) and reveal a mechanism, which we believe is novel, for endocannabinoid secretion into the intestinal lumen. Knockdown or inhibition of $\mathrm{P}$-gp reduced luminal secretion levels of $\mathrm{N}$-acyl ethanolamine-type endocannabinoids, which correlated with increased neutrophil transmigration in vitro and in vivo. Additionally, loss of CB2, the peripheral cannabinoid receptor, led to increased pathology and neutrophil influx in models of acute intestinal inflammation. These results define a key role for epithelial cells in balancing the constitutive secretion of antiinflammatory lipids with the stimulated secretion of proinflammatory lipids via surface efflux pumps in order to control neutrophil infiltration into the intestinal lumen and maintain homeostasis in the healthy intestine.
\end{abstract}

\section{Introduction}

The healthy intestine is remarkable for the absence of an inflammatory response in the presence of dense microbial colonization. Maintenance of homeostasis requires a dynamic, regulated balance among the host immune response, resident microbiota, and the thin epithelial layer that separates them. While maintaining a state of noninflammation, however, the immune system must remain poised to mount an inflammatory response upon exposure to pathogens. Dysregulation of this balance can have serious consequences that may drive a variety of pathological conditions, including inflammatory bowel disease (IBD) (1). Epithelial cells serve as a physical barrier to microbial penetration and provide a sentinel system to warn immune cells of pathogen exposure by secreting neutrophil chemoattractants, placing them in a critical position to regulate the balance between pro- and antiinflammatory states. One of the first events associated with induction of a proinflammatory state is the transepithelial migration of neutrophils from the submucosal compartment to the lumen of the epithelium: a 1-way

\section{Related Commentary: p.3750}

Authorship note: RJM and BAM contributed equally to this work. Conflict of interest: RJM and BAM are coinventors on a patent application (PGT/US 18/42116) emanating from the findings described herein. They, along with their respective academic institutions, stand to gain financially through potential commercialization outcomes resulting from activities associated with the licensing of that intellectual property. Submitted: August 10, 2017; Accepted: June 19, 2018.

Reference information: J Clin Invest. 2018;128(9):4044-4056.

https://doi.org/10.1172/JCI96817. cellular-trafficking process that allows neutrophils to engage with pathogens before they can enter the host.

Neutrophil recruitment to access the epithelial lumen must occur via a carefully regulated multistep cascade of events, as the powerful effectors released by recruited neutrophils potently kill bacteria but lack specificity and thus can also cause severe collateral tissue damage. Basolateral release of IL- 8 by epithelial cells stimulates recruitment of neutrophils out of the vasculature and into the lamina propria underlying the epithelial layer (1). However, IL-8 is unable to mediate the final step, in which neutrophils migrate from the basolateral (serosal) surface of the epithelium to its apical (luminal) surface. We previously identified an innate immune pathway in intestinal epithelial cells that functions to establish the chemotactic gradient required for neutrophils to move from the submucosal space to the lumen of the gut, where subsequent activation leads to inflammatory outcomes. We defined a critical role in this process for the eicosanoid hepoxilin $\mathrm{A}_{3}\left(\mathrm{HxA}_{3}\right)(2)$, a potent neutrophil chemoattractant that is secreted from the apical surface of the epithelium via the multi-drug resistance protein 2 (MRP2) efflux pump (3). Polarized expression of MRP2 and the activity of this $\mathrm{MRP} 2 / \mathrm{HxA}_{3}$ axis are greatly increased during inflammatory states, and this pathway is nonredundant with other chemoattractants driving neutrophil recruitment, such as IL-8. Additionally, we have shown that the MRP2/HxA 3 axis is a conserved mechanism at mucosal surfaces and can drive a damaging inflammatory response with severe pathological consequences (3-11). Clinical evidence further indicates that the $\mathrm{MRP} 2 / \mathrm{HxA}_{3}$ pathway is acti- 
A

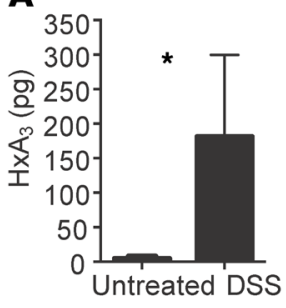

B

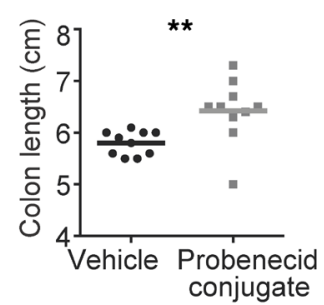

C

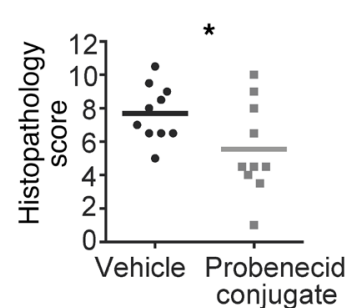

D

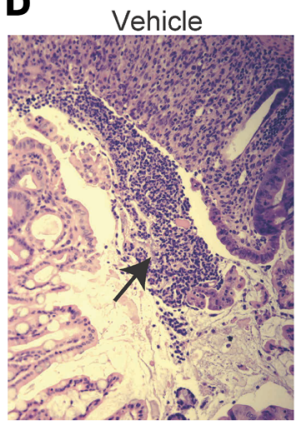

Probenecid

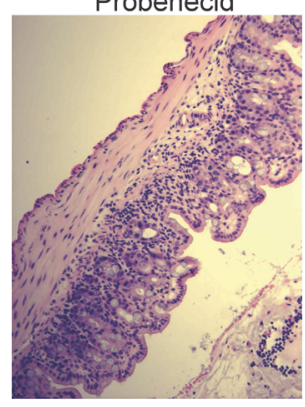

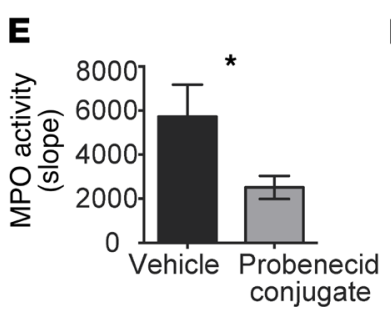

$\mathbf{F}$

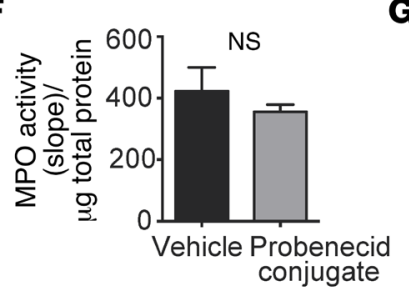

G

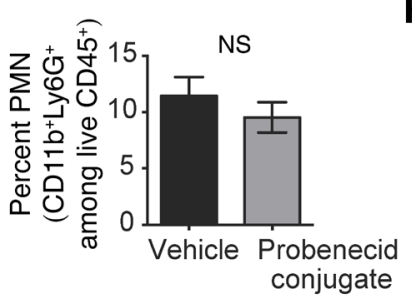

H

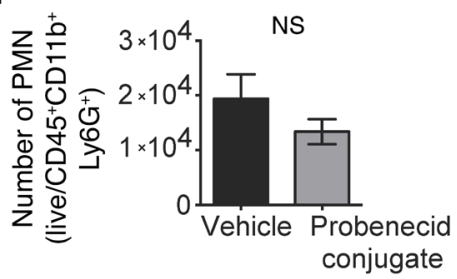

I

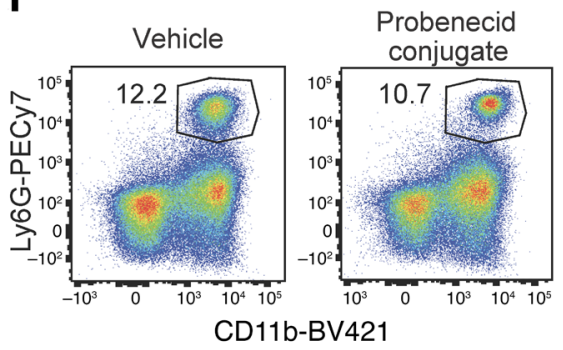

Figure 1. HxA3 drives inflammation during DSS colitis. (A) Mucosal scrapings from C57BL/6 WT mice $(n=10)$ treated with $3 \%$ DSS for 7 days were enriched for lipids, and the amount of HxA3 was quantified by LC-MS/MS. ${ }^{*} P=0.050$. (B-I) C57BL/ 6 mice were treated with $3 \%$ DSS for 7 days and sacrificed at day 9 . Starting at day 4, daily rectal administration of PBS control (vehicle) or $1 \mathrm{mM}$ probenecid conjugate was performed. ${ }^{*} P=0.001$, Mann-Whitney 1-tailed nonparametric $U$ test. All data are shown as mean \pm SEM. $n=10$ mice per group. (C and D) Paraffin-embedded sections of mid and distal colon were stained for $\mathrm{H} \& \mathrm{E}$ and scored by a trained investigator blinded to sample identity. ${ }^{*} P=0.026$. Arrow highlights accumulation of neutrophils in intestinal lumen. Original magnification, $\times 20$. (E and F) MPO activity was measured by ADHP assay over 8 minutes from $(\mathbf{E})$ feces $\left({ }^{*} P=0.044\right)$ or $(\mathbf{F})$ colonic tissue and slopes calculated by linear regression $(P=0.362)$. For tissue, slopes were normalized to total protein content. (G-I) Total lamina propria leukocytes were isolated and stained for flow cytometry. Neutrophils were characterized as live/ CD45 ${ }^{+}$CD11 b hi Ly6C $^{+}$. (G) Percentage of neutrophils (NS, $P=0.193$ ), (H) number of neutrophils (NS, $P=0.259)$, and (I) representative plots of neutrophils in colon tissue. vated in ulcerative colitis (UC) and Crohn disease (CD) patients (3), possibly due to aberrant regulation of signaling pathways.

Transepithelial migration is specifically associated with diminished barrier function, and while neutrophils in the lumen may kill pathogenic microbes, their sustained activity can cause intestinal damage. In fact, disruption of the final transmigration step in mice results in the basolateral collection of neutrophils without apparent damage, emphasizing the critical nature of transepithelial migration in neutrophil-driven pathology (12). We hypothesize that the $\mathrm{MRP} 2 / \mathrm{HxA}_{3}$ axis forms the proinflammatory arm of a dynamically regulated system in which inflammatory pathways that activate responses to pathogens or aberrant signals are balanced against antiinflammatory pathways that suppress neutrophil responses in the context of normal commensal colonization. We therefore investigated the mechanisms by which epithelial cells regulate transepithelial neutrophil migration during homeostasis and inflammation.

\section{Results}

We previously observed that epithelial cells dramatically increase surface expression of the membrane ABC transporter MRP2 in response to infection with a variety of pathogens including Salmonella typhimurium (3). Concurrent with this increased surface MRP2 expression, the intracellular biosynthetic pathway of the proinflammatory eicosanoid $\mathrm{HxA}_{3}$ is upregulated (13). We then demonstrated that MRP2 transports $\mathrm{HxA}_{3}$ into the intestinal lumen, establishing a chemotactic gradient biased toward the lumen that directs migration of neutrophils from the basolateral space across the epithelium, a critical step in the inflammatory process $(2,3)$. Having shown that this MRP2/ $\mathrm{HxA}_{3}$ pathway is conserved during infection with multiple pathogens in both lung and intestinal epithelia $(5,7,14)$, we sought to determine whether it also drives inflammation in the absence of infection; certain forms of IBD as well as several other clinically important human diseases (e.g., rheumatoid arthritis, psoriasis) are characterized by excessive neutrophilic inflammation with no known infectious agent.

Treatment of mice with dextran sodium sulfate (DSS) induces acute colonic inflammation characterized by epithelial damage and neutrophil influx (15). Semiquantitative liquid chromatographydual mass spectrometry (LC-MS/MS) analysis of colonic mucosal scrapings revealed that DSS treatment strongly induced secretion of $\mathrm{HxA}_{3}$ at the epithelial surface (Figure 1A). To confirm that this increased $\mathrm{HxA}_{3}$ contributes to disease, $\mathrm{HxA}_{3}$ secretion was specifically blocked via inhibition of MRP2 using probenecid (3). We chemically conjugated probenecid to periodate-oxidized $40 \mathrm{kDa}$ dextran through a reductive amidation reaction to produce a high molecular weight, hydrophilic material that would be essentially restricted to the apical surface of the intestine following its intrarectal admin- 
A

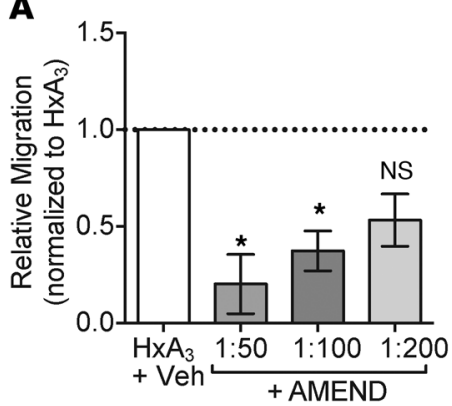

C

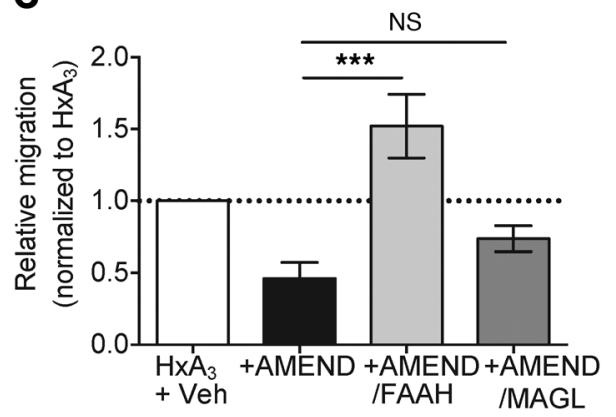

B

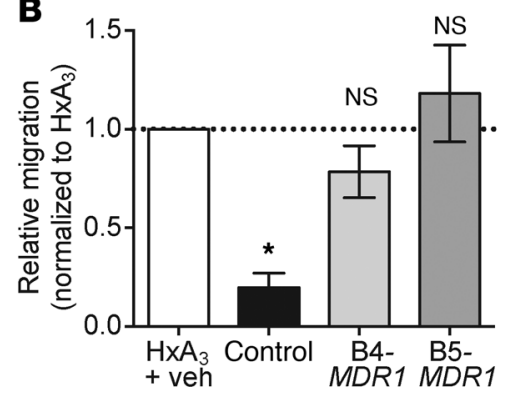

D

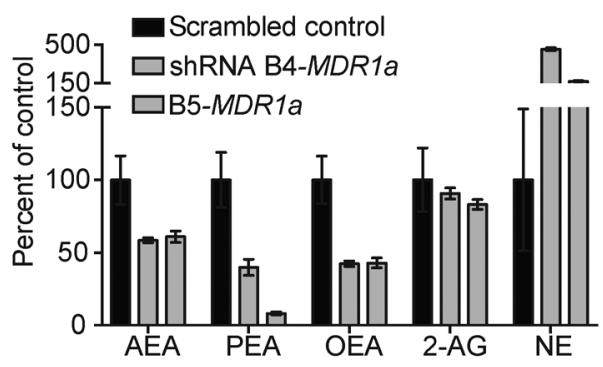

Figure 2. Epithelial cells secrete P-gp-dependent ECs that inhibit neutrophil migration. (A) Supernatants from T84 epithelial monolayers were enriched for lipids and tested for the ability to inhibit HxA3-induced migration in a 96-well modified Boyden chamber assay. In order to compare across experiments with different donors, migration values within individual experiments were normalized to enriched $\mathrm{HxA} 3$ with vehicle treatment. For $\mathbf{A}-\mathbf{C}$, data are shown as mean \pm SEM of 3 independent experiments. ${ }^{*} P<0.05:{ }^{* *} P$ $<0.01,1$-way ANOVA. (B) Performed as in A, with supernatants from cell lines expressing different shRNA constructs to knock down P-gp expression (B4-MDR1 and B5-MDR1). (C) Performed as in A, but prior to use in the migration assays, enriched T84 supernatants were pretreated with FAAH or MAGL at $37^{\circ} \mathrm{C}$ for 30 minutes. (D) Lipid-extracted T84 supernatants from control or 2 separate MDR1-knockdown cell lines (B4 and B5) were subjected to electrospray ionization MS. AEA-d8 was included in each sample to allow quantitative comparisons between samples and was used to calculate relative units of each EC. Individual ECs were normalized to the relative units in the scrambled control condition.

istration in order to target luminal MRP2 and minimize systemic exposure. We confirmed that this probenecid-dextran conjugate was functional in vitro in inhibiting neutrophil migration in a Salmonella infection assay (Supplemental Figure 1; supplemental material available online with this article; https://doi.org/10.1172/ JCI96817DS1). In vivo, inhibition of the MRP2/HxA pathway by intrarectal administration of the probenecid-dextran conjugate significantly reduced intestinal pathology and colon shortening induced by DSS (Figure 1, B-D); though not shown, the histopathology score of mice without DSS was measured as 0. Analysis of colon histopathology revealed that mice treated with the probenecid-dextran conjugate had reduced neutrophil infiltration into the colonic lumen (Figure 1, C and D; the vehicle cohort had a histopathology score of 8.5 , whereas the probenecid conjugate had a histopathology score of 4.5), which was confirmed by a significant reduction in fecal sample myeloperoxidase (MPO) levels (Figure 1E). Conversely, quantitation of neutrophils in the lamina propria during DSS-induced influx revealed no significant difference with probenecid treatment (Figure $1, \mathrm{~F}-\mathrm{I})$, indicating that treatment primarily blocked migration across the epithelium into the lumen. These findings are consistent with other studies of the MRP2/ $\mathrm{HxA}_{3}$ pathway and suggest that neutrophil transepithelial migration is particularly important in exacerbating inflammatory pathology.
In parallel with increased MRP2 levels, another luminal ABC transporter restricted to the luminal epithelial surface, P-glycoprotein (P-gp), is actively reduced at the epithelial surface by Salmonella typhimurium $(16,17)$, the significance of which was previously unclear. P-gp is a multidrug transporter known to efflux small neutral or positively charged hydrophobic compounds (18). Defects in P-gp expression or function are associated with IBD: decreased P-gp expression is observed in the epithelium of IBD patients, and single-nucleotide polymorphisms in the MDR1 gene encoding P-gp are associated with increased IBD risk (19-21). Further, mice lacking the Mdrla gene that encodes P-gp develop spontaneous intestinal inflammation $(22,23)$. Evidence correlating reduced P-gp function with inflammation, combined with the hydrophobic nature of known exogenous P-gp substrates, led us to hypothesize that P-gp might secrete endogenous bioactive lipids that could serve to antagonize $\mathrm{HxA}_{3}$ mediated neutrophil transepithelial migration. We therefore analyzed the P-gp-dependent secreted lipidome of homeostatic epithelial cells to identify lipids capable of inhibiting $\mathrm{HxA}_{3}-$ mediated neutrophil migration.

Supernatants collected from the apical surface of noninflamed T84 colonic epithelial cell monolayers inhibited primary human neutrophil transmigration stimulated by $\mathrm{HxA}_{3}$ in an in vitro assay; we termed the unknown compound(s) in this preparation activity modulating epithelial neutrophil discourse (AMEND). Similar spent media collected from the basal compartment of T84 cell monolayers failed to exhibit AMEND activity. Subsequent size fractionation of crude AMEND preparations using ultrafiltration demonstrated AMEND to be less than $1 \mathrm{kDa}$. An extensive series of BAKERBOND Sep-Pak extraction columns were tested to further characterize the AMEND activity. Outcomes of these matrix interaction studies suggested AMEND as having a lipophilic character (Supplemental Table 1). Notably, AMEND bound strongly to the Narc-2 matrix, used for the capture and elution of active agents in marijuana (24). The resulting optimized AMEND collection protocol involved ultrafiltration of cell supernatants to isolate compounds smaller than $1 \mathrm{kDa}$, followed by lipid enrichment using reversed-phase $\mathrm{C}_{18}$ chromatography, yielding a fraction capable of inhibiting primary human neutrophil migration stimulated by $\mathrm{HxA}_{3}$ in a cell-free in vitro assay (Figure 2A). To confirm that AMEND is specifically secreted by P-gp, we created stable knockdown human T84 epithelial cell lines using shRNA to target MDR1 and confirmed reduction of P-gp expression by Western blot (Supplemental Figure 2). Enriched superna- 

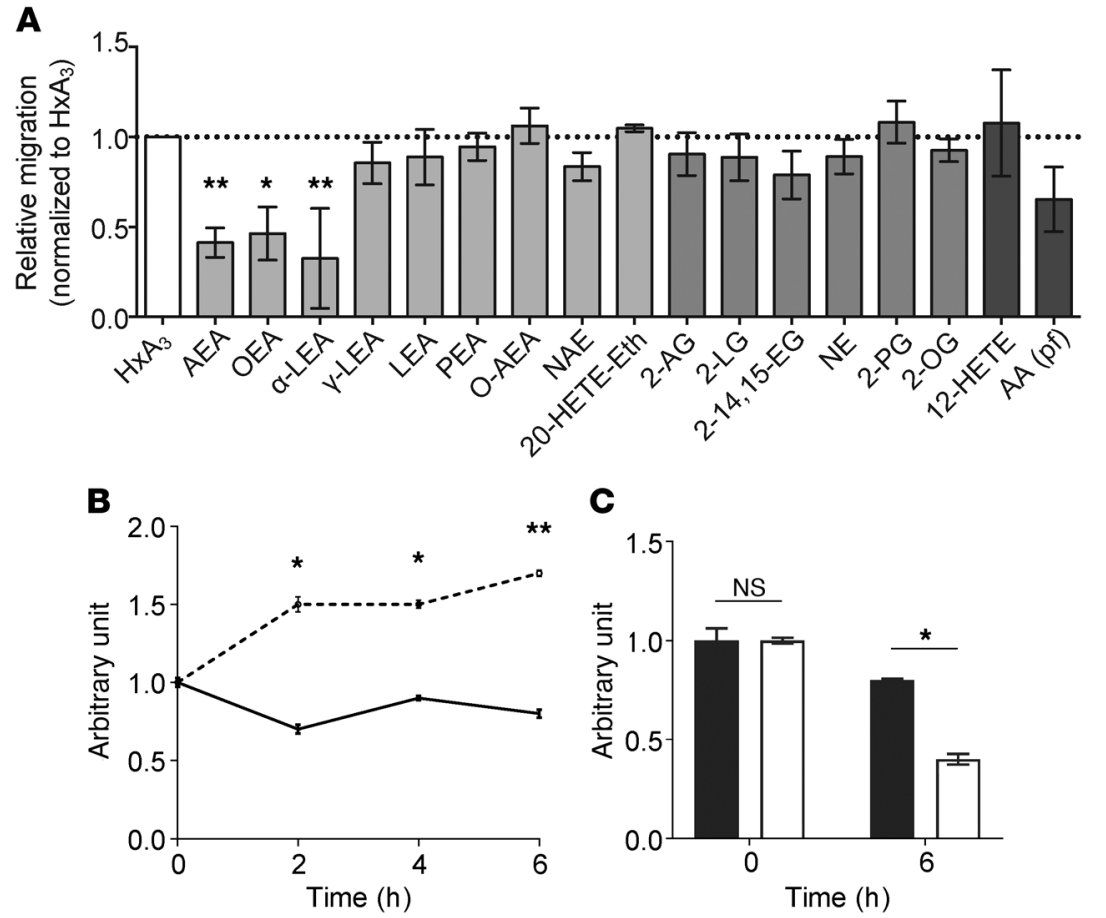

Figure 3. P-gp-dependent apical secretion of ECs inhibits neutrophil migration. (A) Commercially available $E C s$ and related compounds were tested in the 96-well migration assay, with AEA, $O E A$, and $\alpha$-LEA significantly suppressing neutrophil movement. Compounds were used at the highest concentration at which they were soluble in PBS. Data are shown as mean \pm SEM of at least 3 independent experiments. ${ }^{*} P<0.05$; ${ }^{* *} P<0.01$, 1-way ANOVA. (B) Confluent $2 \mathrm{D}$ human intestinal organoid cultures were used to examine apical secretion of AEA over a 6-hour time course with and without $50 \mu \mathrm{M}$ verapamil (apical untreated, apical with verapamil), with levels being reported as average of triplicate measurements $( \pm S D)$ normalized to time 0 values. Increasing apical compartment levels of AEA over time were suppressed by the presence of $50 \mu \mathrm{M}$ verapamil. Data are shown as mean \pm SD from 3 independent experiments with 12-24 technical replicates in each experiment. ${ }^{*} P<0.01$; ${ }^{* *} P<0.001$, unpaired $t$ test. (C) The levels of AEA present in the basal compartment of confluent $2 \mathrm{D}$ human intestinal organoid cultures remained relatively constant over this same 6 -hour time course, but were reduced in cultures treated with $50 \mu \mathrm{M}$ verapamil. Relative changes in the basal compartment AEA levels were measured at 0 and 6 hours, with (white bars) and without (black bars) $50 \mu \mathrm{M}$ verapamil addition. AEA levels are reported as normalized to time 0 values. Data are shown as mean \pm SD from 3 independent experiments with $12-24$ technical replicates in each experiment. ${ }^{*} P<0.01$, unpaired $t$ test.

tants from P-gp-deficient cells lacked AMEND activity and failed to inhibit neutrophil migration (Figure 2B). Similar results (Supplemental Figure 3) were obtained following treatment of WT T84 cells with verapamil, an inhibitor of P-gp (25).

To identify AMEND components responsible for inhibition of neutrophil transmigration, we first undertook a target-oriented approach to probe for receptor activation. Enriched AMEND was screened for both agonist and antagonist activity against a GPCR panel, using an assay for $\beta$-arrestin activity that is independent of $G$ protein subtype (26). Consistent with its role as an inhibitor of neutrophil transmigration, AMEND displayed primarily antagonist activity at GPCRs, including several known chemoattractant receptors (Supplemental Table 2). Importantly, the strongest signal observed was for agonist activity at CB2, the peripheral cannabinoid receptor, suggesting that AMEND is composed of one or more endocannabinoid (EC) lipids.

ECs are defined by their activity at the classical cannabinoid receptors $\mathrm{CB} 1$ and $\mathrm{CB} 2$ as well as more recently described atypical receptors GPR55 and GPR119 $(27,28)$. Potential interplay between these cannabinoid receptors is complex and the functional roles of each in the context of intestinal inflammation are still poorly understood $(29,30)$. The 2 main classes of eicosanoid-type ECs are $\mathrm{N}$-acyl ethanolamines (NAEs) and monoacylglycerols (MAGs), which are metabolized by fatty acid amide hydrolase (FAAH) and monoacylglycerol lipase (MAGL), respectively $(31,32)$. To determine whether AMEND activity is provided by ECs, we examined its sensitivity to these enzymes. Treatment of enriched AMEND with FAAH completely eliminated the inhibitory activity of AMEND, whereas treatment with MAGL did not significantly affect the ability of AMEND to inhibit $\mathrm{HxA}_{3}$-induced migration (Figure $2 \mathrm{C}$ ), suggesting that AMEND belongs to the NAE class. To confirm the specificity of enzyme treatment, we performed a similar experiment with the migrationinhibiting non-EC lipid lipoxin $\mathrm{A}_{4}$, which was not sensitive to deactivation by FAAH or MAGL (Supplemental Figure 4).

We performed MS to identify AMEND components secreted from the apical epithelial surface in a P-gp-dependent manner. First, luminal secretions were collected from polarized monolayers of T84 cells with and without verapamil treatment to block P-gp function. Unique peaks, identified by comparison of peak profiles of luminal contents of these 2 treatments separated using $\mathrm{C} 18$ reversed phase highperformance LC protocol, were found to have masses consistent with the $\mathrm{H}^{+}$and $\mathrm{Na}^{+}$adduct masses of known ECs, specifically, anandamide (AEA) (Supplemental Figure 5). We next performed a semiquantitative MS analysis, comparing supernatants obtained from control and MDR1knockdown P-gp-deficient T84 intestinal epithelial cells. Samples were spiked with deuterated AEA- $\mathrm{d}_{8}$ as an internal MS standard prior to enrichment of AMEND activity by ultrafiltration and reversed phase $\mathrm{C}_{18}$ chromatography as above prior to being subjected to high-resolution MS. $\mathrm{H}^{+}$and $\mathrm{Na}^{+}$adduct masses consistent with AEA, oleoyl ethanolamide (OEA), and palmitoyl ethanolamide (PEA) were reduced in the absence of P-gp expression (Figure 2D and Supplemental Table 3). These ECs all belong to the NAE class; other lipids of the MAG class of ECs, including 2-arachidonoyl glycerol (2-AG) and noladin ether (NE), were present at similar or even increased amounts in the absence of P-gp (Figure 2D and Supplemental Table 3). These results are consistent with AMEND being enzymatically susceptible to FAAH more than MAGL and support the hypothesis that P-gp specifically secretes NAE-class ECs.

We next tested purified ECs in the in vitro $\mathrm{HxA}_{3}$-induced neutrophil migration assay. Owing to the possibility that additional EC species were present in the original AMEND material, but not detected by MS analysis, a wide panel of commercially available purified ECs were tested. Only AEA, OEA, and $\alpha$-linolenoyl 
A

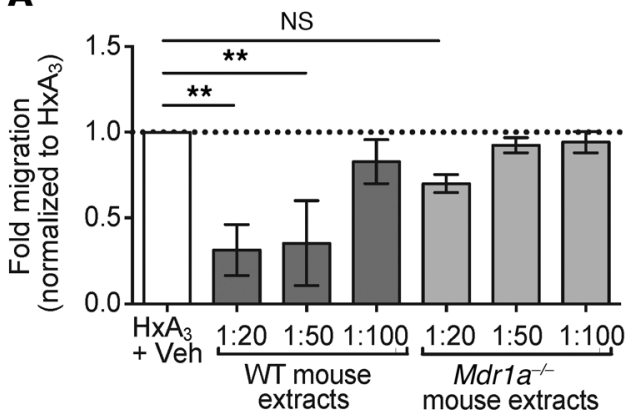

B

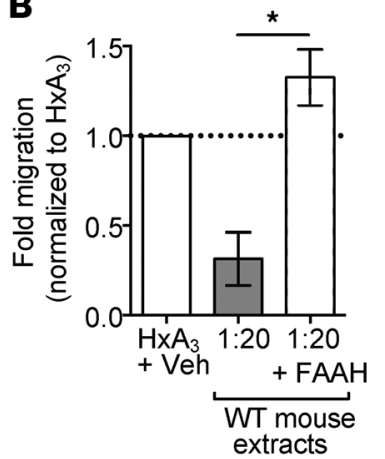

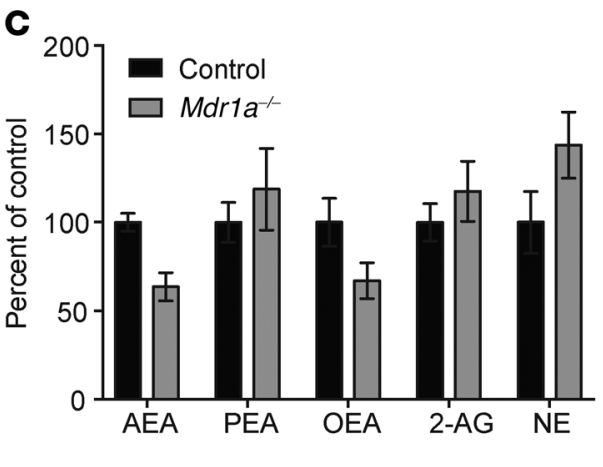

Figure 4. AMEND is present in mouse intestine. (A) Colonic scrapings from 5 WT or Mdr1a ${ }^{-/-}$FVB mice were pooled, enriched for lipids, and tested in the 96-well migration assay as in Figure 2. Data are shown as mean \pm SEM from 3 independent experiments. ${ }^{* *} P<0.01$, 1-way ANOVA. (B) Indicated sample was pretreated with FAAH for 30 minutes at $37^{\circ} \mathrm{C}$. ${ }^{*} P=0.01$. (C) Intestinal mucosal scrapings from WT and Mdr1a-/- FVB mice were lipid extracted (modified Folch method) and analyzed by MS. Mice were pooled in groups of 3 . Data shown are from 3 pooled groups. Semiquantitative analysis was performed as in Figure 2.

ethanolamide ( $\alpha$-LEA) exhibited significant inhibitory activity, identifying these NAEs as putative AMEND components (Figure 3A). While other EC types have been reported as affecting neutrophil function in vitro $(33,34)$, we conclude that only NAE-type ECs are capable of specifically inhibiting $\mathrm{HxA}_{3}$-mediated neutrophil migration. Additional experiments were performed using mixtures of synthetic AEA, OEA, and $\alpha$-LEA to determine their role in CB2-related effects of AMEND. Unsurprisingly, we found that these NAE-type ECs, when tested individually as well as in combinations, failed to directly act as $\mathrm{CB} 2$ receptor agonists; only AEA showed some activity and this was approximately 10 -fold less active than 2-AG (Supplemental Table 4). Consistent with this observation, we screened for CB2 activity using NADA (a potent endogenous cannabinoid and vallinoid receptor agonist) and GP1a (a selective CB2 receptor agonist) as well as JTE 907 (a selective CB2 receptor inverse agonist); only JTE 907 showed signs of activity (Supplemental Figure 6). Taken together, these experiments reveal the possibility that AEA, OEA, and $\alpha$-LEA may not function as direct $\mathrm{CB} 2$ receptor agonists, but rather act indirectly to regulate $\mathrm{CB} 2$ function, highlighting the complexity of their role or roles in inhibiting $\mathrm{HxA}_{3}$-mediated neutrophil migration. Thus, further investigations are required to reveal the nature of such intricate pharmacological interactions.

Since our studies have focused on how inflammatory signals regulate the transmigration of neutrophils from the basolateral compartment to the intestinal lumen, we hypothesize that such a system requires a biased release of agents to establish a chemical gradient from the apical epithelial surface. In support of this hypothesis, we have previously shown that the MRP2 transport pump involved in regulating the efflux of $\mathrm{HxA}_{3}$ from the apical surface is restricted to this cell region to allow the establishment of such a gradient (2). Indeed, this prior work used human intestinal xenographs to show that the biology we are focusing on should be present in humans in vivo. To therefore extend the clinical significance of these findings herein, we chose to follow the pattern of AEA release, as the AMEND prototype, within cell supernatants collected from the apical or basolateral compartment of healthy human-derived organoid (colonoid) epithelial monolayers grown
A

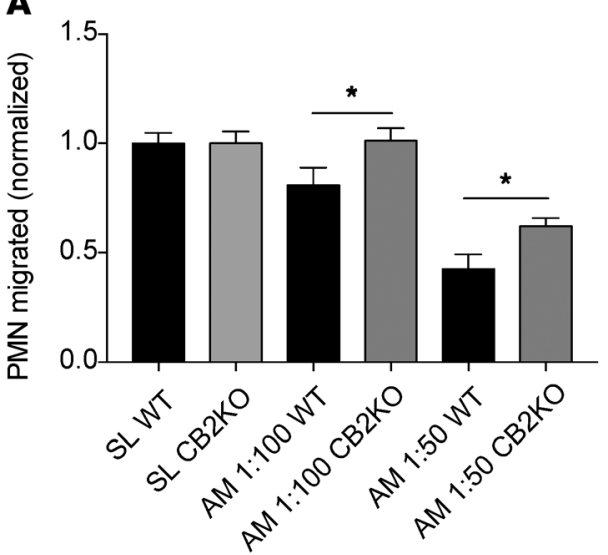

B

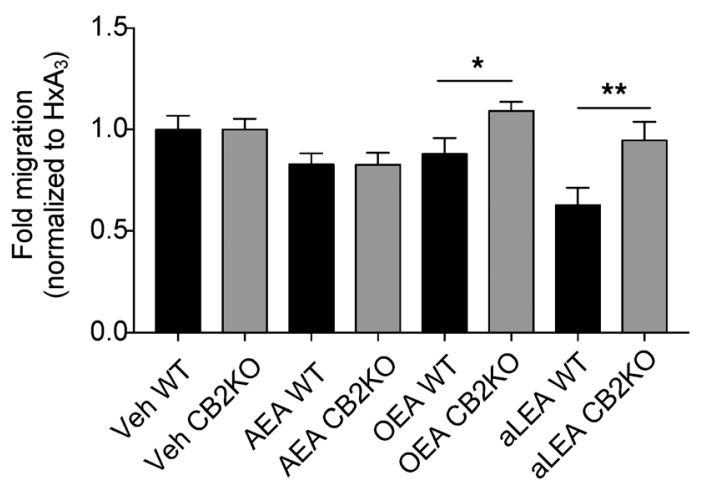

Figure 5. Signaling through CB2 contributes to AMEND inhibition of neutrophil migration. Neutrophils were isolated from the BM of WT or $\mathrm{Cnr}^{-1-}$ (CB2KO) mice and allowed to migrate across Salmonella-infected epithelial monolayers. $\mathrm{SL}$, Salmonella. (A) Neutrophil migration was tested in the presence of enriched AMEND (AM). ${ }^{*} P<0.05$. (B) Neutrophil migration was tested in the presence of purified ECs. ${ }^{*} P<0.05$; ${ }^{*} P<0.01$. Data are shown as mean \pm SD from 3 independent experiments. Comparison of groups was performed by Mann-Whitney nonparametric $U$ test.

in a $2 \mathrm{D}$ format (see Methods) in the absence or presence of the P-gp inhibitor verapamil. The apical and basal compartments of the 2D colonoid monolayers were exposed to verapamil as a standard method to inhibit the efflux of substrates by P-gp that is selectively expressed at the apical surface of intestinal epithelial cells.

As shown in Figure 3B, the relative concentration of AEA increased in the apical compartment of the 2D colonoid cultures over the 6-hour study time course, but this increase was blocked by verapamil. However, AEA present in the basal compartment at the 


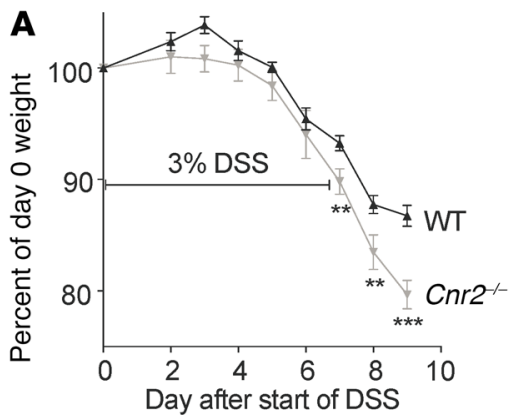

C
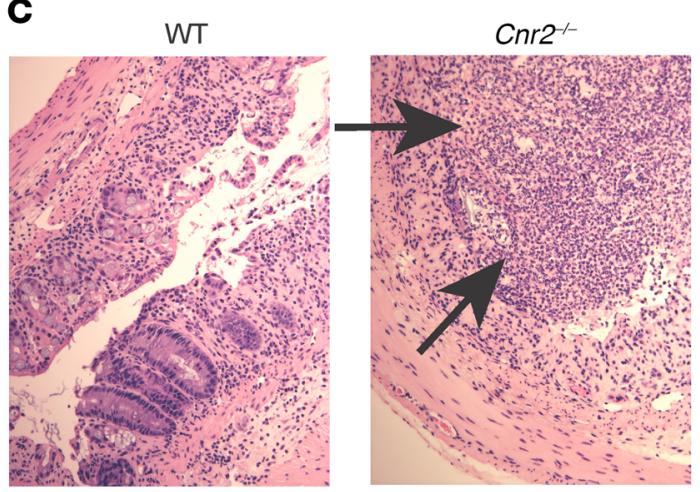

$\mathbf{F}$

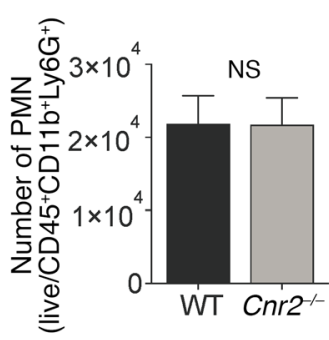

G
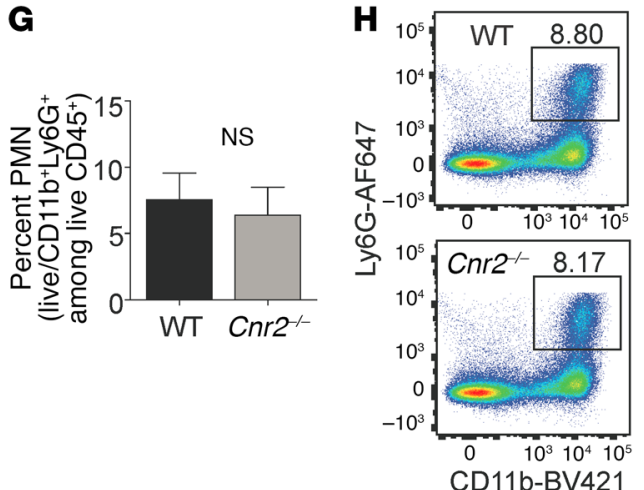

B

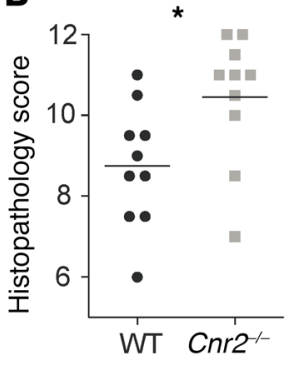

D

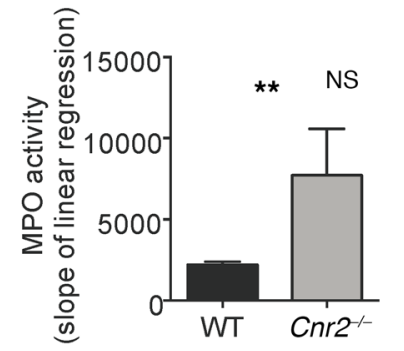

E

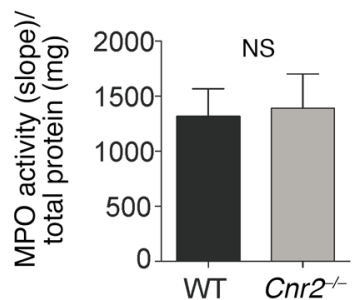

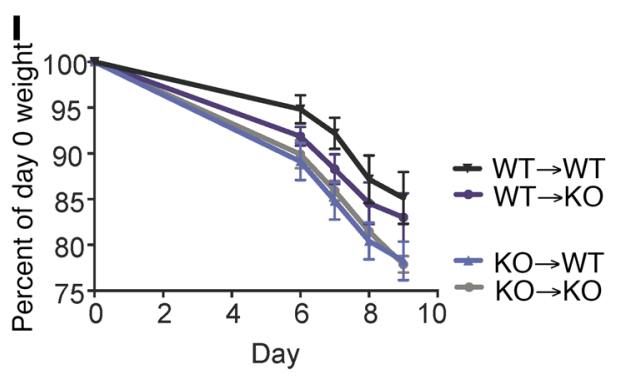
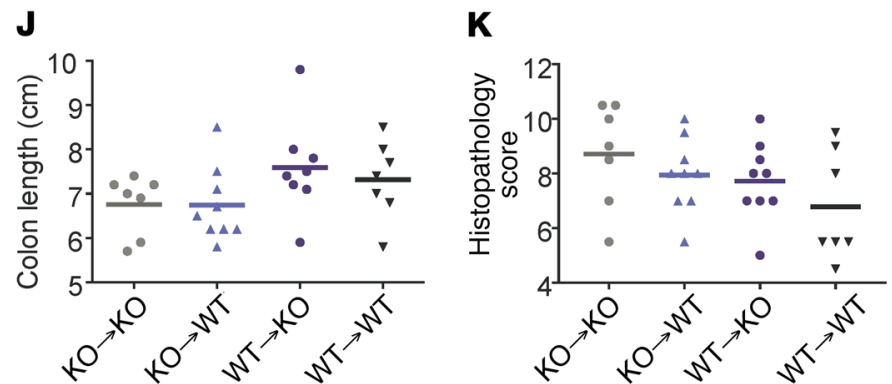

Figure 6. CB2-deficient mice are vulnerable to severe intestinal inflammation with increased neutrophil transmigration. WT or Cnr2 ${ }^{-/-}$mice were treated with $3 \%$ DSS for 7 days and sacrificed at day 9 . (A) Weights are shown as percentage of day 0 (DO) weight. $n=25$ WT; $n=24$ Cnr2 ${ }^{-/-}$mice. ${ }^{* *} P<0.01 ;{ }^{* *} P<$ 0.001. (B and C) Histopathology of mid and distal colon as in Figure 1. Arrows highlight accumulation of neutrophils in intestinal lumen. ${ }^{*} P=0.021$. Original

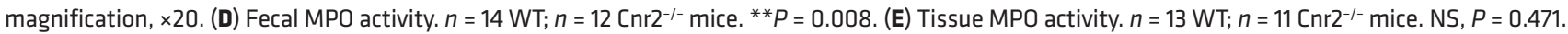
(F) Number (NS, $P=0.482$ ) and (G) percentage (NS, $P=0.095$ ) of tissue neutrophils by flow cytometry analysis. (H) Representative plots of lamina propria neutrophils. (F-H) $n=13 \mathrm{WT} ; n=12 \mathrm{Cnr}^{-1-}$ mice. (I-K) WT or $\mathrm{Cnr} 2^{-1-}$ mice were lethally irradiated and reconstituted with homologous or heterologous BM (donor $\rightarrow$ recipient). Following reconstitution for 7 weeks, mice were treated with DSS as above. (I) Weight curve. (J) Colon length. (K) Histopathology score. $n=7 \mathrm{WT} \rightarrow \mathrm{WT} ; n=9 \mathrm{KO} \rightarrow \mathrm{WT} ; n=9 \mathrm{WT} \rightarrow \mathrm{KO} ; n=7 \mathrm{KO} \rightarrow \mathrm{KO}$ mice. For all experiments, data are shown as mean \pm SEM. Statistical analysis was performed with Mann-Whitney 1-tailed nonparametric $U$ test. Histopathology scoring, MPO activity measurement, and flow cytometry analyses were performed as in Figure 1.

beginning and end of this 6-hour time did not change, although such levels were reduced in 2D cultures treated with verapamil (Figure 3C). The constant level of AEA over time in the basal supernatants suggests that the amount of AEA reaching the basal compartment is dependent on paracellular diffusion from the apical compartment and not an efflux from the basolateral surface of these 2D cultures, as we failed to observe an increase in AEA levels over this time period. Thus, there appears to be a balance between the rate of consumption and/or degradation of AEA at the basolateral surface of enterocytes and the egress of AEA from the apical compartment. Reduction in basal AEA levels in the verapamil-treated cultures likely reflects the reduced input rate to AEA via the paracellular route from the apical compartment without a coordinated change in the rate of consumption and/or deg- radation of AEA. Overall, these results are consistent with efflux of AEA by apically expressed P-gp, with its subsequent redistribution across the epithelium to suppress transmigration of neutrophils by direct interaction with these cells. Levels of 2-AG in the apical and basal compartment of this 2D monolayer model of healthy human intestine were below the level of detection.

A selective mechanism for EC transport out of cells had not been previously identified, although a putative membrane protein capable of bidirectional transport of AEA and 2-AG has been hypothesized (35). Having now established P-gp as an apical cell membrane efflux pump that exports NAE-type ECs in cultured epithelial cells, we sought to confirm this pathway in the intestine. Mucosal scrapings from the colons of WT and P-gp deficient $\left(M_{d r 1 a^{-/}}\right)$mice were enriched for AMEND and evaluated for their 


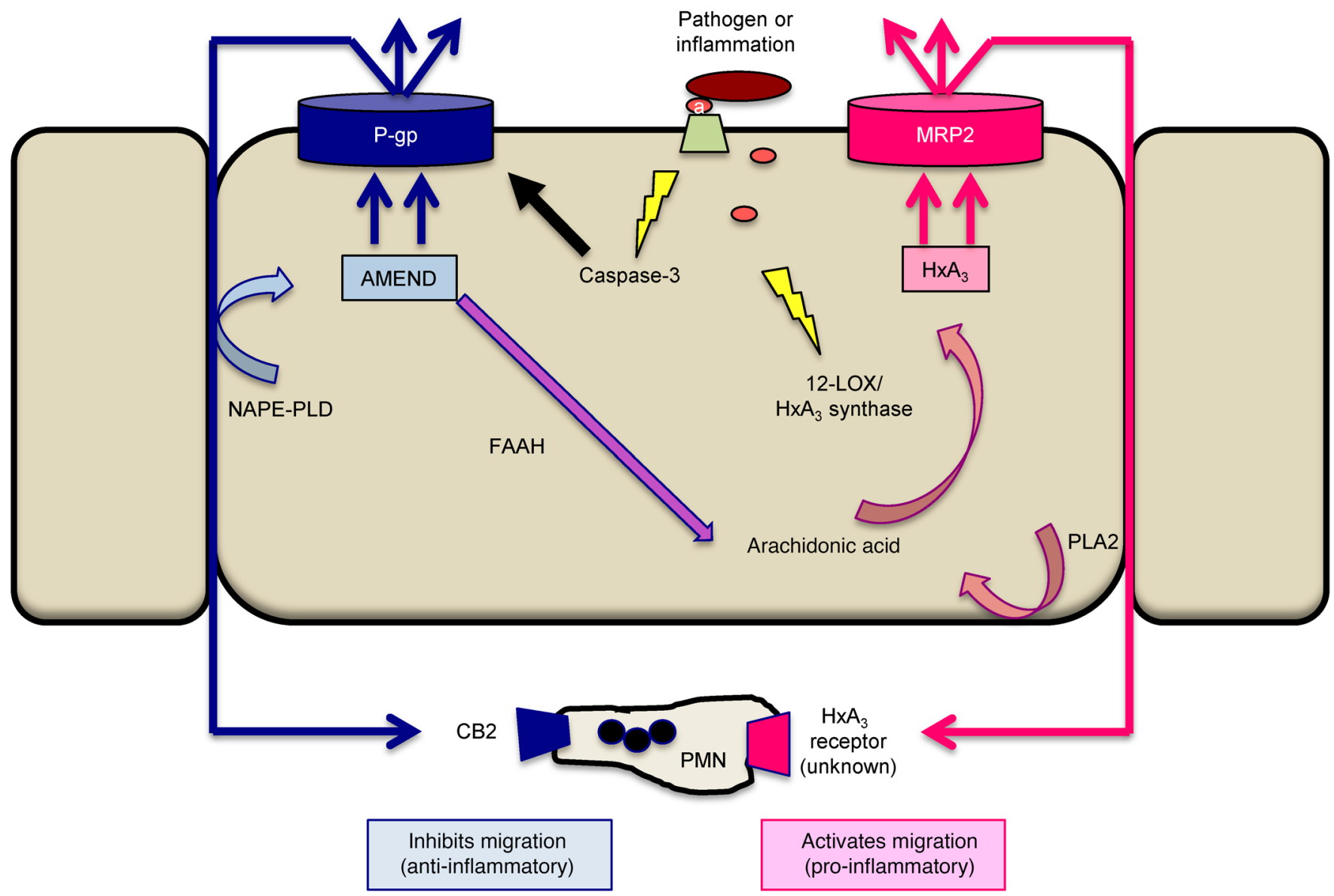

Figure 7. The antiinflammatory P-gp/EC and proinflammatory MRP2/HxA3 pathways in the intestinal epithelium. In the homeostatic intestine, $\mathrm{P}$-gp secretes ECs from the epithelial surface. Secreted NAEs act through the CB2 receptor on neutrophils to inhibit migration and maintain an antiinflammatory state. During inflammation, P-gp is downregulated and the MRP2/HxA3 pathway is activated. Phospholipase A2 liberates arachidonic acid from the membrane, and it is converted to HxA3 and secreted into the lumen via surface MRP2. HxA3 forms a concentration gradient that attracts neutrophils across the epithelial layer into the lumen, where they cause inflammatory damage and pathology. Of note, FAAH metabolism of NAEs yields arachidonic acid and may also feed into the proinflammatory MRP2/HXA3 pathway.

ability to inhibit neutrophil migration. These ex vivo scrapings from WT mice inhibited in vitro neutrophil transmigration similarly to AMEND isolated from T84 cells, whereas scrapings from $M d r 1 a^{-1-}$ mice lacked inhibitory AMEND activity (Figure 4A). When scrapings from WT mice were pretreated with FAAH, they lost their inhibitory activity, confirming that these samples contained NAE ECs (Figure 4B). High-resolution MS revealed that similar NAEtype ECs correlated with the presence of P-gp in vitro (Figure 2D) and in vivo (Figure 4C) with the exception of PEA, which was equally abundant in samples obtained from both $M d r 1 a^{-/-}$and WT mice (Figure 4C and Supplemental Table 5). Although $\alpha$-LEA was identified as an AMEND candidate in Figure 3B, it is not included in the LIPID MAPS database used for MS analysis, and so its potential dependence on P-gp export remains unknown.

The peripheral cannabinoid receptor $\mathrm{CB} 2$, which is expressed on immune cells, was identified as a potential AMEND target in our GPCR screen (Supplemental Table 2). While 2-AG is the canonical CB2 ligand, 2-AG, AEA, and other NAE-type ECs can activate $\mathrm{CB} 1, \mathrm{CB} 2$, and other non-CB receptors under varying conditions $(36,37)$. Additionally, mixed EC populations may have more complex effects, including the development of agonists or antagonists/inverse agonists when combined with other ECs (38-40). In order to determine whether CB2 signaling is required for inhibition of neutrophil migration by AMEND, we isolated neutrophils from WT and CB2-deficient $\left(\mathrm{Cnr}^{-/-}\right)$mice. Migration of $\mathrm{Cnr}^{-/-}$neutrophils across Salmonella-infected epithelial monolayers was significantly less impaired compared with WT neutrophil migration in the presence of enriched AMEND (Figure 5A). However, at higher concentrations, AMEND was able to partially inhibit migration of $\mathrm{Cnr}^{-/-}$neutrophils, suggesting that signaling through receptors in addition to $\mathrm{CB} 2$ may contribute to AMEND activity. When experiments were performed with purified AEA, OEA, and $\alpha$-LEA, CB2 expression contributed to inhibition of neutrophil transmigration by OEA and $\alpha$-LEA, but not AEA (Figure $5 \mathrm{~B}$ ), although mouse neutrophils in this assay were less susceptible to AEA inhibition for reasons we cannot yet explain.

We next investigated the potential for P-gp-mediated secretion of NAE-type ECs to functionally suppress $\mathrm{HxA}_{3}$-mediated intestinal inflammation in vivo. In keeping with previous reports $(23,41)$, $M d r 1 a^{-/-}$mice, which would lack the capacity for P-gp-dependent EC secretion, developed spontaneous intestinal inflammation with age (data not shown) and were more susceptible to DSS-induced colitis 
(Supplemental Figure 7). CB2 agonists have been demonstrated to provide beneficial effects in colitis (42-44), and we hypothesized that CB2-deficient mice would be less able to respond to the P-gpsecreted inhibitory NAE-type ECs. In support of this hypothesis, Cnr2 $2^{---}$mice treated with DSS were more vulnerable to inflammation and developed increased intestinal pathology and weight loss compared with WT mice (Figure 6, A-C). In particular, Cnr2-- mice displayed dramatic increases in neutrophil influx into the intestinal lumen (Figure 6, C and D), while neutrophil accumulation in the tissue was similar in both groups (Figure 6, E-H). As was observed during blockade of normal neutrophil transepithelial migration during DSS-induced colitis using probenecid to inhibit MRP2 (Figure 1), neutrophil migration specifically across the epithelium into the lumen was associated with significant tissue damage that contributed independently to disease pathology (Figure 6). The enhanced susceptibility of $\mathrm{Mdrla} \mathrm{a}^{-/}$and $\mathrm{Cnr} 2^{-/-}$mice to intestinal inflammation suggests that absence of a functional P-gp/EC-signaling pathway perturbs homeostasis, leading to increased vulnerability to onset and possibly maintenance of an inflammatory state.

The development of inflammation in $\mathrm{MdrIa}^{-/-}$mice depends specifically on the absence of P-gp in the epithelial compartment (22), consistent with our model in which epithelial P-gp export of NAE-type ECs blocks inflammation. In order to confirm that the increased susceptibility of $\mathrm{Cnr}^{-/-}$mice to intestinal inflammation resulted from a failure of neutrophils to respond to EC inhibition, we created heterologous $\mathrm{BM}$ chimeras and evaluated the relative contributions of CB2 in the immune and nonimmune compartments to susceptibility to DSS-induced colitis. Overall, mice transplanted with $\mathrm{Cnr}^{-/-} \mathrm{BM}$, regardless of recipient genotype, displayed the greatest weight loss and colon shortening, indicative of increased inflammation (Figure 6, I and J). Transfer of WT BM into $\mathrm{Cnr} 2^{-/-}$recipients partially abrogated the inflammatory phenotype, as these heterologous mice developed less inflammation than seen in transfer of $\mathrm{Cnr}^{-/-} \mathrm{BM}$ donors into $\mathrm{Cnr}^{-/-}$recipient controls (Figure $6, \mathrm{I}-\mathrm{K})$. While this experiment did not fully rule out a potential role for CB2 in epithelial or other stromal cells, the data are consistent with our hypothesis in suggesting that CB2 expression on radiation-sensitive immune cells plays a dominant role in preventing development of colitis via inhibitory signaling by ECs.

In sum, these studies have defined an antiinflammatory P-gp/EC pathway that acts to counterbalance the proinflammatory $\mathrm{MRP} 2 / \mathrm{HxA}_{3}$ axis. We propose that balancing the P-gp/EC and $\mathrm{MRP} 2 / \mathrm{HxA}_{3}$ axes is a mechanism by which epithelial cells themselves integrate signals from the local environment to maintain exquisite control over the initiation of neutrophil-mediated inflammation. While multidrug-resistant transporters are generally studied for their role in exporting drugs and foreign compounds from the cell, our results indicate that these efflux pumps expressed at the apical surface of intestinal epithelial cells provide a critical link in communication between sentinel functions of mucosal barriers and the immune system. In fact, by exporting bioactive lipids to regulate neutrophil transepithelial migration, MRP2 and P-gp uniquely control a critical step of the inflammatory cascade (Figure 7). It should be noted that the polarized organization of the P-gp/ $\mathrm{EC}$ and MRP2/ $\mathrm{HxA}_{3}$ axes at the apical surface of intestinal epithelial cells is presumed to be critical for either suppressing or stimulating neutrophil transepithelial migration. This apically restricted organization of P-gp/EC to maintain homeostatic conditions can be rapidly reversed by apical inflammatory signal(s) through processes involving $\mathrm{MRP} 2 / \mathrm{HxA}_{3}$ that are independent of neutrophil function in the submucosal compartment.

\section{Discussion}

Identification of a functional P-gp/EC antiinflammatory pathway that opposes the proinflammatory $\mathrm{MRP} 2 / \mathrm{HxA}_{3}$ axis and controls neutrophil transmigration provides a potential explanation for several poorly understood biological and clinical observations. P-gp was originally identified as a xenobiotic efflux pump (45), and no endogenous substrates have heretofore been identified. Previous in vitro studies examining the impact of AEA on P-gp function were interpreted as inhibiting the function of this efflux pump (46), but these data are consistent with the possibility that AEA can function as a P-gp substrate. We now show NAE-type ECs to be endogenous substrates for P-gp. Our finding that antiinflammatory NAE secretion from epithelial cells depends on P-gp also provides a potential mechanism underlying both the development of spontaneous intestinal inflammation in P-gp-deficient mice (22) and the clinical evidence that P-gp dysfunction contributes to IBD (20).

Previous studies have demonstrated suppression of colitis symptoms by CB2 agonists (42-44); demonstration of P-gp secretion of NAE-type ECs that can suppress neutrophil transepithelial migration, coupled with the observation that CB2deficient mice demonstrated an increase in this same process, mechanistically links these findings to a specific role for NAEtype ECs and CB receptor function during intestinal disease. Previous studies have suggested that ECs or CB2 ligands can reduce neutrophil migration $(33,34)$, but the same studies also showed induction of neutrophil migration by AEA and 2-AG (34), leaving the true function in vivo an open question (47). We did not observe any chemoattractant activity of ECs alone; all of our studies were consistent with inhibition of neutrophil transepithelial migration in the colon by NAE-type ECs that functioned at least partially via CB2 agonism. The present results may also provide a possible mechanistic explanation for anecdotal data from colitis patients suggesting clinical benefits from exposure to cannabinoids (48).

The present studies have identified a new layer of regulation of neutrophil function in the intestine. Unlike other antiinflammatory lipids, such as the specialized proresolving mediators that are produced by immune cells (49), ECs appear to be secreted locally at the apical surface by epithelial cells and can therefore constitutively maintain an antiinflammatory environment during intestinal homeostasis. Modulation of $\mathrm{MRP} 2 / \mathrm{HxA}_{3}$ and $\mathrm{P}-\mathrm{gp} / \mathrm{EC}$ function at the luminal surface of the intestine represents an exciting new avenue for development of topical therapeutics for the treatment of IBD. The MRP2/ $\mathrm{HxA}_{3}$ pathway is conserved in infectious and noninfectious lung inflammation $(4,9,50)$, suggesting that the P-gp/EC pathway may similarly regulate inflammation at other mucosal surfaces. Additionally, other neutrophil-like cell types, such as granulocytic myeloid-derived suppressor cells involved in $\mathrm{T}$ cell tolerance, have also been shown to be involved in inflammatory events associated with IBD (51). It will be interesting to examine the potential for the P-gp/EC and MRP2/ $\mathrm{HxA}_{3}$ axes described herein to regulate their actions. 


\section{Methods}

Human cell lines. T84 intestinal epithelial cells at passages 50-79 (ATCC) were grown in a mixture of DMEM and Ham's F12 Nutrient Mixture (Thermo Fisher Scientific) supplemented with $14 \mathrm{mM} \mathrm{NaHCO}_{3}, 15 \mathrm{mM}$ HEPES buffer ( $\mathrm{pH} 7.5$ ), $40 \mathrm{mg} / \mathrm{l}$ penicillin, $8 \mathrm{mg} / \mathrm{l}$ ampicillin, $90 \mathrm{mg} /$ 1 streptomycin, and $5 \%$ heat-inactivated FBS. HCT- 8 colon carcinoma cells (ATCC) and H292 lung epithelial carcinoma cells (CRL 18-48; ATCC) were grown in RPMI 1640 with $10 \%$ heat-inactivated FBS.

Salmonella infection of HCT-8 epithelial cells. HCT-8 cell monolayers were grown and maintained on inverted $0.33 \mathrm{~cm}^{2}$ ring-supported, collagen-coated $5 \mu \mathrm{m}$ pore polycarbonate filters or 96-well HTS Transwell filter plates (Costar Corp.). Cells were treated apically and basolaterally with probenecid conjugate at $100 \mu \mathrm{M}$ in $\mathrm{HBSS}$ with $\mathrm{Ca}^{2+}$ and $\mathrm{Mg}^{2+}\left(\mathrm{HBSS}^{+/+}\right)$incubated for 1 hour at $37^{\circ} \mathrm{C}$. Following washing, cells were infected apically with Salmonella enterica serovar typhimurium strain SL1344 (SL) at an MOI of 375 for 1 hour. Following extensive washing, neutrophils $\left(1 \times 10^{6}\right)$ were added to the basolateral surface, allowed to transmigrate across the monolayer for 2 hours, and quantified as described below.

Production of enriched $\mathrm{HxA}_{3}$. The extraction protocol and purity of $\mathrm{HxA}_{3}$ have been previously reported by the authors of this report $(2,4$, 5). Briefly, to induce $\mathrm{HxA}_{3}$ efflux, Pseudomonas aeruginosa strain PAO1 was grown aerobically in Luria broth (LB) broth overnight at $37^{\circ} \mathrm{C}$. Cultures were washed once in $\mathrm{HBSS}^{+/+}$and suspended at a concentration of $6 \times 10^{7}$ bacteria/ml. H292 monolayers in $162 \mathrm{~cm}^{2}$ flasks were infected for 1 hour, washed with HBSS, and then incubated in $\mathrm{HBSS}^{+/+}$ for 5 hours. Collected supernatants were captured by reversed phase chromatography on octadecylsilane $\left(\mathrm{C}_{18}\right)$ columns (Supelco; SigmaAldrich), washed with water, and eluted with methanol. Samples were stored at $-20^{\circ} \mathrm{C}$ and the volume necessary for individual experiments dried down and suspended in $\mathrm{HBSS}^{+/+}$as needed. Each new batch of enriched $\mathrm{HxA}_{3}$ was quality tested before use in experiments and was generally used after a 1:4 to 1:8 dilution.

Generation of P-gp knockdown T84 cell lines. shRNA constructs targeting the MDR1a gene were generated from a pLKO.1 plasmid background and the DNA sequences used were derived from the TRC-Hs 1.0 (human) shRNA library, which is publicly available and searchable based on the TRCN identifier at the Genetic Perturbation Platform (https://portals.broadinstitute.org/gpp/public/clone/ search). Constructs are from the TRC-Hs 1.0 (human) shRNA library and are publicly available, constructs were as follows: B4 (clone TRCN0000059683), B5 (clone TRCN0000059684), B6 (clone TRCN0000059685), B7 (clone TRCN0000059686), and B8 (clone TRCNO000059687). Lentiviruses were produced by transfecting packaging cells (293T) with psPAX2, pMD.2G, and pLK0.1 plasmid constructs, using Trans-IT-LT1 lipid (Mirus Bio). After 48 hours, lentiviral supernatants were harvested, combined with $8 \mu \mathrm{g} / \mathrm{ml}$ polybrene (Sigma-Aldrich), and applied to T84 cells in 20\% confluent monolayers. This process was repeated 24 hours later, and 48 hours following the second transfection, resistant cells were selected with $5 \mu \mathrm{g} / \mathrm{ml}$ puromycin. Once stably transfected lines were obtained, reduction of P-gp expression was confirmed by Western blot using anti-P-gp monoclonal antibody C219 (catalog 517310, EMD Millipore).

Production of enriched AMEND. T84 cells were grown as confluent monolayers in $162 \mathrm{~cm}^{2}$ flasks and equilibrated in $\mathrm{HBSS}^{+/+}$at $37^{\circ} \mathrm{C}, 5 \%$ $\mathrm{CO}_{2}$. For verapamil treatment of cells, $40 \mu \mathrm{M}$ verapamil hydrochloride (MilliporeSigma) was included during the entire incubation. Cell super- natants collected over the course of 5 hours were pooled from 30 flasks, lyophilized, suspended in water, and ultrafiltered through an Amicon 1,000 Da cutoff membrane (MilliporeSigma) with $\mathrm{N}_{2}$-positive pressure. Samples were desalted and captured on $\mathrm{a}_{18}$ Bakerbond SepPak column that was washed with water and then hexane prior to elution with methanol, dried under $\mathrm{N}_{2}$ gas, and stored at $-80^{\circ} \mathrm{C}$. Before use in migration experiments, samples were suspended in $\mathrm{HBSS}^{+/+}$as needed. Individual batches of AMEND were tested for activity in control assays before experimental use and were generally used at a 1:100 to 1:200 final dilution. For screening in the DiscoveRx GPCR $\beta$-arrestin activity assay, samples were suspended in PBS to provide a $\times 1,000$ solution for screening.

Preparation of the human organoid-derived epithelial monolayers. Human intestinal epithelial cells were obtained from patients undergoing medically required surgical resections as determined by a licensed physician, as described previously $(52,53)$, with modifications. The present studies used cells isolated from healthy margins, as verified by a pathologist, of tissue resected from the ascending colon to obtain intestinal crypts. After washing once in ice-cold $1 \times$ PBS (Thermo Fisher Scientific), tissue strips were cut and incubated at $4^{\circ} \mathrm{C}$ for 30 minutes in a dissociation buffer consisting of $1 \times$ PBS, penicillin/ streptomycin (Thermo Fisher Scientific), $1 \mathrm{mM}$ dithiothreitol (SigmaAldrich), and 0.5 mM EDTA (Sigma-Aldrich). Tissue samples were then vigorously shaken to promote epithelial cell dissociation from the basement membrane, with this procedure being repeated 5 times to collect multiple fractions containing intestinal crypts that were further processed and then plated in Matrigel as previously described (53). Intestinal crypt-derived organoids were incubated in humidified $5 \%$ $\mathrm{CO}_{2}$ at $37^{\circ} \mathrm{C}$ in media that consisted of a $1: 1$ ratio of stem cell media and L-WRN-derived (ATCC CRL-3276) conditioned media (54). Culture medium was replenished every other day, with organoids being passaged every 7 to 9 days using standard trypsin-based techniques. Approximately $2.0 \times 10^{6}$ cells $/ \mathrm{ml}$ were plated in Matrigel to ensure robust organoid propagation.

Single-cell suspensions derived from organoids were plated at 1.0 $\times 10^{6}$ cells $/ \mathrm{ml}$ on polyethylene terephthalate membrane Transwell inserts having a $0.4 \mu \mathrm{m}$ pore size (Corning) and incubated in 1:1 stem cell/L-WRN media at $37^{\circ} \mathrm{C}$ with $5 \% \mathrm{CO}_{2}$ (55). Culture medium was changed every other day until confluence was reached as determined by transepithelial electrical resistance (TEER) monitoring and microscopic observation. At 48 hours prior to each experiment, the basolateral media were replenished with fresh 1:1 stem cell/L-WRN media. Medium in the apical chamber was replaced with complete DMEM plus $5 \mu \mathrm{M}$ of the $\gamma$-secretase inhibitor IX (DAPT; Calbiochem) to promote cell differentiation (55). On the day of each experiment, monolayers were washed with $1 \times$ PBS and both the apical and basolateral media were replaced with DMEM lacking phenol red; monolayers were incubated for at least 2 hours before initiation of an experiment.

Collection of AMEND from human organoid-derived epithelialmonolayers. Confluent organoid monolayers grown on polyethylene terephthalate membrane Transwell inserts in 24-well plates were washed and equilibrated in $\mathrm{HBSS}^{+/+}$at $37^{\circ} \mathrm{C}, 5 \% \mathrm{CO}_{2}$ for 30 minutes. At the end of the equilibration step, supernatants from the apical and basolateral compartments were collected and stored at $4^{\circ} \mathrm{C}$ as the 0 hours samples. Liquid was replaced in both apical and basolateral compartments with $50 \mu \mathrm{M}$ verapamil in $0.5 \% \mathrm{DMSO} / \mathrm{HBSS}^{+/+}$in treated wells or $\mathrm{DMSO} / \mathrm{HBSS}^{+/+}$alone in control wells. Verapamil was added to both the apical and basal chambers, since this molecule would equilibrate 
in such a closed system. Supernatants from the apical compartment were collected and replaced with $0.5 \% \mathrm{DMSO} / \mathrm{HBSS}^{+/+}$with or without $50 \mu \mathrm{M}$ verapamil every 2 hours up to 6 hours to monitor the efflux of AMEND. To assess accumulation of AMEND in the basolateral chamber of this model, supernatants from the basolateral compartment were collected and replaced with $0.5 \% \mathrm{DMSO} / \mathrm{HBSS}^{+/+}$with or without $50 \mu \mathrm{M}$ verapamil at 0 and 6 hours. Cells were maintained at $37^{\circ} \mathrm{C}, 5 \% \mathrm{CO}_{2}$ throughout the experiment. Samples were pooled from replicate wells for each compartment, apical and basolateral, and centrifuged at $1,452 \mathrm{~g}$ for 15 minutes at $4^{\circ} \mathrm{C}$ to remove cell debris. Supernatants were stored at $-20^{\circ} \mathrm{C}$ prior to LC/MS analysis of AMEND. Data are shown as mean \pm SD from 3 independent experiments with 12-24 technical replicates in each experiment. TEER values (mean \pm SD) for each independent experiment are as follows: (a) $492.91 \pm 88.7$; (b) $441.01 \pm 45.87$; and (c) $447.91 \pm 19.93$.

96-Well neutrophil migration. Peripheral blood neutrophils from healthy human volunteers were purified from acid-citrate-dextrose anticoagulated peripheral blood by $2 \%$ gelatin sedimentation, as previously described (14). Red blood cells were removed by lysis in cold $\mathrm{NH}_{4} \mathrm{Cl}$ buffer, and neutrophils were washed with $\mathrm{HBSS}^{-/-}$(without $\mathrm{Ca}^{2+}$ or $\mathrm{Mg}^{2+}$ ) and suspended to a final volume of $5 \times 10^{7} / \mathrm{ml}$. 96-Well HTS Transwell filter plates (Corning), $3 \mu \mathrm{m}$ pore size, were coated with $0.1 \mathrm{mg} / \mathrm{ml}$ rat tail collagen and allowed to dry overnight. Enriched $\mathrm{HxA}_{3}$ (see above) was added to the lower compartment along with a final 1:10 dilution of vehicle control, enriched AMEND (see above), or purified EC compounds at the indicated concentrations. Neutrophils $\left(5 \times 10^{5}\right)$ were added to the top well along with a final 1:10 dilution of vehicle or purified ECs, placed in a $37^{\circ} \mathrm{C}$ incubator with $5 \%$ $\mathrm{CO}_{2}$, and allowed to migrate for 2 hours. Top wells were removed, and transmigrated neutrophils were lysed with $1 \%$ Triton $\mathrm{X}-100$. Sodium citrate buffer (0.1 M, pH 4.2) was added, and an equal volume of 2,2'azino-bis (3-ethylbenzothiazoline-6-sulphonic acid) (ABTS) in $0.1 \mathrm{M}$ sodium citrate was added to samples. MPO activity was measured, and neutrophil cell equivalents were calculated by comparison with a standard curve, with data from individual experiments being normalized to $100 \% \mathrm{HxA}_{3}$-driven migration. Data are shown as mean \pm SEM from at least 3 independent experiments. Statistical analysis was performed using GraphPad Prism; data were analyzed by either 1-way ANOVA or Mann-Whitney nonparametric $U$ test, as appropriate for experimental conditions.

Purified compounds used in migration assays. All compounds were obtained from Cayman Chemical and suspended at the highest concentration at which they were soluble in PBS (based on the manufacturer's instruction and empirical observation) and then diluted 1:10 to final concentrations as indicated:

Arachidonoyl ethanolamide (AEA), Chemical Abstracts Service (CAS) no. 94421-68-8, was used at a final concentration of $0.01 \mathrm{mg} / \mathrm{ml}$. $\alpha$-LEA, CAS no. 57086-93-8, was used at $0.005 \mathrm{mg} / \mathrm{ml}$. LEA, CAS no. 68171-52-8, was used at $0.0001 \mathrm{mg} / \mathrm{ml} . \gamma$-LEA, CAS no. 150314-37-7, was used at $0.0001 \mathrm{mg} / \mathrm{ml}$. 2-AG, CAS no. 53847-30-6, was used at 0.01 $\mathrm{mg} / \mathrm{ml}$. 2-Linoleoyl glycerol (2-LG), CAS no. 3443-82-1, was used at 0.01 $\mathrm{mg} / \mathrm{ml}$. 2-(14,15-Epoxyeicosatrienoyl) glycerol, CAS no. 848667-56-1, was used at $0.005 \mathrm{mg} / \mathrm{ml}$. NAE, CAS no. 94421-69-9, was used at 0.005 $\mathrm{mg} / \mathrm{ml}$. O-Arachidonoyl ethanolamine (O-AEA), CAS no. 443129-35-9, was used at $0.001 \mathrm{mg} / \mathrm{ml}$. NE, CAS no. 222723-55-9, was used at 0.001 $\mathrm{mg} / \mathrm{ml}$. 12-Hydroxyeicosatetraenoic acid (12-HETE), CAS no. 71030$37-0$, was used at $0.005 \mathrm{mg} / \mathrm{ml}$. 20-HETE ethanolamide (20-HETE
Eth), CAS No 942069-11-6, was used at $0.005 \mathrm{mg} / \mathrm{ml}$. OEA, CAS no. 111-58-0, was used at $0.01 \mathrm{mg} / \mathrm{ml}$. 2-Palmitoyl glycerol (2-PG), CAS no. 23470-00-0, was used at $0.05 \mathrm{mg} / \mathrm{ml}$. 2-Oleoyl glycerol (2-OG), CAS no. 3443-84-3, was used at $0.002 \mathrm{mg} / \mathrm{ml}$. PEA, CAS no. 544-31-0, was used at $0.0000005 \mathrm{mg} / \mathrm{ml}$. Arachidonic acid, peroxide-free formulation (pfAA), CAS no. 506-32-1, was used at $0.125 \mathrm{mg} / \mathrm{ml}$. NADA (a potent endogenous cannabinoid and vallinoid receptor agonist), GP1a (a selective CB2 receptor agonist), and JTE 907 (a selective CB2 receptor inverse agonist), were purchased from Tocris Bioscience.

LC/MS analysis of AMEND. MeOH-eluted preparations as above were dried under a stream of $\mathrm{N}_{2}(\mathrm{~g})$, suspended in $\mathrm{MeOH} /$ buffer, and separated by HPLC using a Vydac (Hichrom) C18 (10 um; 300 Å) semi-preparative column $(10 \times 250 \mathrm{~cm})$. Active AMEND fractions were characterized by HPLC/MS (Genesis C18 $(4 \mu \mathrm{m}, 120 \AA$ ) analytical HPLC column $(4.6 \times 150 \mathrm{~mm})$ equilibrated with $5 \mathrm{mM}$ triethylamine acetic acid ( $\mathrm{pH}$ 7.2), with the effluent analyzed by using a Finnigan LCQ Deca electrospray mass spectrometer.

Selected samples were analyzed for high mass accuracy determination and solubilized in 50:50 acetonitrile $/ \mathrm{H}_{2} \mathrm{O}$ with $0.2 \%$ formic acid. A MAXIS-HD ultra-high resolution quadrupole time-of-flight (UHR-ESI-QTOF) mass spectrometer (Bruker Daltonik GmbH) was used; this was coupled to a syringe driver (Hamilton) with the sample solution being infused at a rate of $3 \mu \mathrm{l} / \mathrm{min}$.

Nitrogen acted as the nebulizing gas, applied at a pressure of 2 bar. The drying gas was also nitrogen, supplied at a flow rate of $8 \mathrm{l} /$ min and a temperature of $200^{\circ} \mathrm{C}$. Positive ion mode was used with a corresponding capillary voltage of $-4500 \mathrm{~V}$. Only full scan data were acquired. For each batch of samples, a solution of $5 \mathrm{mM}$ sodium formate clusters was also analyzed. This acted as an external data file calibrant over the mass range $75-1000 \mathrm{~m} / \mathrm{z}$. The recalibrated detected mass and isotope pattern were used in the FindFormula tool to generate a list of potential theoretical formulae within $2 \mathrm{mDa}$ of the detected mass. The detected isotope pattern was used to sort this list.

Data acquisition and automated processing were controlled via Compass OpenAccess 1.7 software (Bruker Daltonik GmbH), and data processing was carried out using DataAnalysis 3.4 (Bruker Daltonik $\mathrm{GmbH})$. At each step, care was taken to avoid sample degradation and oxidation by maintaining samples on ice and under $\mathrm{N}_{2}$ gas as much as possible. Relative quantification by ion intensities was performed by the summation of intensities for protonated and sodiated ions to compensate for possible variation in adduct formation due to varying biological sodium levels.

Preparation of dextran conjugates. Dextran $(40 \mathrm{kDa})$ was oxidized with $\mathrm{NaIO}_{4}$ in $0.1 \mathrm{M}$ sodium acetate ( $\mathrm{pH}$ 5.0). After dialysis against $\mathrm{H}_{2} \mathrm{O}$, the oxidized dextran was reacted with excess 1,6,-diaminohexane and coupled via reductive amination at $\mathrm{pH} 9.5$ by the addition of sodium borohydride. After a second dialysis against $\mathrm{H}_{2} \mathrm{O}$, reaction with 1fluoro-2,4-dinitrobenzene was used to determine the presence of approximately 21 free $\mathrm{NH}_{2}$ groups per molecule of diaminohexane-modified dextran. $N$-(3-dimethylamino propyl)- $N$ '-ethylcarbodiimide $\mathrm{HCl}$ was used to covalently couple probenecid (11/dextran molecule) or benzoic acid (13/dextran molecule), with the products being dialyzed against water, lyophilized, and stored at $-20^{\circ} \mathrm{C}$.

Mouse experiments. C57BL/6 WT and Cnr2 ${ }^{-/-}$mice were purchased from Jackson Laboratories; FVB WT and $M d r 1 a^{-/-}$mice were purchased from Taconic. Female mice were used at age 6 to 12 weeks, and genotypes were mixed for 2 to 4 weeks prior to experiments to equalize the 
microbiota. Mice were treated with 3\% DSS (molecular weight 36,00050,000; MP Biomedicals) in the drinking water for 7 days, then placed back on normal water and sacrificed at day 9 , which represented peak disease state. Samples from mid and distal colon were fixed in 10\% formalin, paraffin-embedded, sectioned, and stained for histopathological analysis with H\&E. Each sample was graded semiquantitatively from 0 to 3 for 4 criteria: (a) degree of epithelial hyperplasia and goblet cell depletion; (b) leukocyte infiltration in the lamina propria; (c) area of tissue affected; and (d) presence of markers of severe inflammation, such as crypt abscesses, submucosal inflammation, and ulcers. Samples were scored by a trained investigator blinded to sample identity, and mid and distal colon values were averaged to give a colon histopathology score.

Isolation of neutrophils from mouse BM. Neutrophils were isolated from femurs and tibias as described in Mócsai et al. (56), with the following modifications: neutrophils were isolated in HBSS/HEPES without FCS, and red blood cell lysis was performed with ACK lysis buffer for 4.5 minutes. A total of $1 \times 10^{6}$ mouse neutrophils were added to the top well of a 96-well migration plate and allowed to migrate in the basolateral to apical direction across Salmonella-infected HCT8 epithelial cells. Neutrophil migration was quantified by ABTS assay as above.

Creation of BM chimeras. C57BL/6 WT and Cnr2-- mice were lethally irradiated (90-100 Gy) and reconstituted with a total of $10^{7}$ DONOR BM cells from C57BL/6 WT or $\mathrm{Cnr}^{-1-}$. Mice were allowed to reconstitute for 7 weeks before induction of DSS colitis. Approximately 85\%-90\% reconstitution of donor BM was achieved in this experiment.

Isolation of lamina propria leukocytes and flow cytometry. Cell suspensions from the lamina propria were prepared as described previously (57). Intestinal tissue was cut into small pieces, treated with RPMI with $10 \%$ FBS and 5 mM EDTA to remove epithelial cells, and then incubated with $100 \mathrm{U} / \mathrm{ml}$ Collagenase Type VIII (Sigma-Aldrich) for two 1-hour periods. Cells were then applied to a discontinuous $30 \% / 40 \% / 75 \%$ gradient of Percoll (GE Healthcare Life Sciences) and harvested from the $40 \% / 70 \%$ interface. Cells were washed in PBS/0.1\% BSA, incubated with anti-Fc receptor $(\alpha \mathrm{CD} 16 / 32$, catalog 14-0161-81; eBioscience), and stained with Zombie Live/Dead infrared stain (eBioscience), then surface stained with antibodies to CD45 (clone 30-F11, catalog 103130, 103127, 103105), CD11b (clone M1/70, catalog 101236), Ly6G (clone HK1.4, catalog 128025; and clone 127609, catalog 127618), and Ly6C or Gr1 (clone RB6-8C5, catalog 108407). Samples were run on a MACSquant Analyzer 10 (Miltenyi Bioscience) and analyzed using FlowJo software, version 10 (Treestar).

Analysis of MPO content in mouse samples. Samples were assayed for MPO activity as described (56). Tissue sections of colon were frozen in liquid $\mathrm{N}_{2}$ and stored at $-80^{\circ} \mathrm{C}$ until use. Sections were put in hexadecyltrimethylammonium bromide (HTAB) (MilliporeSigma) buffer with lysing matrix D (MP Biomedicals) and homogenized with a FastPrep-24 homogenizer at level 6 for 40 seconds. Samples were combined with ADHP and fluorescence read over 8 minutes. Slopes were calculated by linear regression using Graphpad Prism and normalized to protein content for individual samples as measured by detergenttolerant DC protein assay (Bio-Rad). For analysis of fecal samples, fecal contents were weighed and HTAB buffer added at a ratio of $10 \mu \mathrm{l} / \mathrm{mg}$. Calculated slopes were used directly.
Mass spectrometric analysis of $\mathrm{HXA}_{3}$ in colonic mucosa. C57BL/6 mice were administered $5 \%$ DSS in their drinking water and sacrificed on day 7. The proximal colon from untreated or DSS-treated mice ( 9 mice/cohort) was harvested, and 3 intestinal segments were pooled. Mucosal scrapings were collected by scraping intestinal surfaces with a rubber policeman in PBS, and $\mathrm{HxA}_{3}$ content was analyzed as previously described (17).

Statistics. Data from the DSS studies (Figures 1 and 6) are reported as mean $\pm \operatorname{SEM}, n=10$ mice per group, statistical significance by Mann-Whitney 1-tailed nonparametric $U$ test. Data from the polymorphonuclear leukocyte (PMN) transmigration assays using peripheral human blood neutrophils (Figures 2 and 3A) are reported as mean ratios \pm SEM of 3 independent experiments with statistical significance by 1-way ANOVA. Data from human organoid-derived epithelial monolayers (Figure 3, B and C) are reported as mean \pm SD from 3 independent experiments with 12-24 technical replicates in each experiment, with statistical significance determined by unpaired ( 2 tailed) $t$ test. Experiments assessing the AMEND present in mouse tissue (Figure 4 ) are reported as mean \pm SEM from 3 independent experiments, with statistical significance determined by 1-way ANOVA. Finally, BM migration assays (Figure 5) are reported as mean \pm SD from 3 independent experiments. Comparison of groups was performed by Mann-Whitney nonparametric $U$ test. $P \leq$ 0.05 was considered significant.

Study approval. The care of all animals used in this study and animal experiments performed were in accordance with the University of Massachusetts Medical School IACUC (protocol 1993-12). All neutrophil-based studies were approved by the IRB of the University of Massachusetts Medical School (protocol 13006). Human organoidderived sample collection was approved by the IRB of Massachusetts General Hospital (protocol 2015P001908). All subjects provided informed consent prior to participation in these studies.

\section{Author contributions}

RLS, RJM, and BAM conceived and designed the study. RLS, RJM, CL, AL, CT, ZD, AJO, BX, SR, SEF, CSF, and ALC acquired data. RLS, RJM, AL, ZD, SEF, and BAM analyzed and interpreted data. RLS, RJM, and BAM drafted the manuscript.

\section{Acknowledgments}

We would like to thank Karsten Gronert, Erik Juncker Boll, and Abraham Brass for technical assistance and Lindsey A. Moser, Jeremy C. Jones, and Philip Ahern for critical reading of the manuscript. Stefania Senger from Massachusetts General Hospital is acknowledged for graciously facilitating the human organoid studies. This work was supported by NIH grants R01DK056754 and R01DK109677 (to BAM and RJM) and NIH grants F32 DK098973 and CCFA CDA 410396 (to RLS).

Address correspondence to: Beth A. McCormick, University of Massachusetts Medical School, Department of Microbiology and Physiological Systems, 368 Plantation Street, AS8-2049, Worcester, Massachusetts 01655, USA. Phone: 508.856.6048; Email: beth.mccormick@umassmed.edu.
1. Fournier BM, Parkos CA. The role of neutrophils during intestinal inflammation. Mucosal Immunol. 2012;5(4):354-366.
2. Mrsny RJ, et al. Identification of hepoxilin A3 in inflammatory events: a required role in neutrophil migration across intestinal epithelia. Proc
Natl Acad Sci U S A. 2004;101(19):7421-7426.

3. Pazos M, et al. Multidrug resistance-associated transporter 2 regulates mucosal inflammation by 
facilitating the synthesis of hepoxilin A3. J Immunol. 2008;181(11):8044-8052.

4. Hurley BP, Sin A, McCormick BA. Adhesion molecules involved in hepoxilin A3-mediated neutrophil transepithelial migration. Clin Exp Immunol. 2008;151(2):297-305.

5. Mumy KL, Bien JD, Pazos MA, Gronert K, Hurley $\mathrm{BP}, \mathrm{McC}$ ormick BA. Distinct isoforms of phospholipase A2 mediate the ability of Salmonella enterica serotype typhimurium and Shigella flexneri to induce the transepithelial migration of neutrophils. Infect Immun. 2008;76(8):3614-3627.

6. Boll EJ, et al. The fimbriae of enteroaggregative Escherichia coli induce epithelial inflammation in vitro and in a human intestinal xenograft model. J Infect Dis. 2012;206(5):714-722.

7. Boll EJ, Struve C, Sander A, Demma Z, Krogfelt KA, McCormick BA. Enteroaggregative Escherichia coli promotes transepithelial migration of neutrophils through a conserved 12-lipoxygenase pathway. Cell Microbiol. 2012;14(1):120-132.

8. Tamang DL, et al. Hepoxilin A(3) facilitates neutrophilic breach of lipoxygenaseexpressing airway epithelial barriers. J Immunol. 2012;189(10):4960-4969.

9. Bhowmick R, et al. Systemic disease during Streptococcus pneumoniae acute lung infection requires 12-lipoxygenase-dependent inflammation. J Immunol. 2013;191(10):5115-5123.

10. Agbor TA, Demma Z, Mrsny RJ, Castillo A, Boll EJ, McCormick BA. The oxido-reductase enzyme glutathione peroxidase 4 (GPX4) governs Salmonella Typhimurium-induced neutrophil transepithelial migration. Cell Microbiol. 2014;16(9):1339-1353.

11. Mallick EM, et al. The ability of an attaching and effacing pathogen to trigger localized actin assembly contributes to virulence by promoting mucosal attachment. Cell Microbiol. 2014;16(9):1405-1424.

12. Kucharzik T, et al. Acute induction of human IL-8 production by intestinal epithelium triggers neutrophil infiltration without mucosal injury. Gut. 2005;54(11):1565-1572.

13. Pace-Asciak CR, Reynaud D, Demin P. Mechanistic aspects of hepoxilin biosynthesis. J Lipid Mediat Cell Signal. 1995;12(2-3):307-311.

14. Hurley BP, Siccardi D, Mrsny RJ, McCormick BA. Polymorphonuclear cell transmigration induced by Pseudomonas aeruginosa requires the eicosanoid hepoxilin A3. JImmunol. 2004;173(9):5712-5720.

15. Okayasu I, Hatakeyama S, Yamada M, Ohkusa $\mathrm{T}$, Inagaki $Y$, Nakaya R. A novel method in the induction of reliable experimental acute and chronic ulcerative colitis in mice. Gastroenterology. 1990;98(3):694-702.

16. Siccardi D, Mumy KL, Wall DM, Bien JD, McCormick BA. Salmonella enterica serovar Typhimurium modulates P-glycoprotein in the intestinal epithelium. Am J Physiol Gastrointest Liver Physiol. 2008;294(6):G1392-G1400.

17. Mercado-Lubo R, et al. A Salmonella nanoparticle mimic overcomes multidrug resistance in tumours. Nat Commun. 2016;7:12225.

18. Siarheyeva A, Lopez JJ, Glaubitz C. Localization of multidrug transporter substrates within model membranes. Biochemistry. 2006;45(19):6203-6211.
19. Brant SR, et al. MDR1 Ala893 polymorphism is associated with inflammatory bowel disease. $A m$ JHum Genet. 2003;73(6):1282-1292.

20. Ho GT, Gaya DR, Satsangi J. Multidrug resistance (MDR1) gene in inflammatory bowel disease: a key player? Inflamm Bowel Dis. 2005;11(11):1013-1019.

21. Brinar M, et al. MDR1 polymorphisms are associated with inflammatory bowel disease in a cohort of Croatian IBD patients. BMC Gastroenterol. 2013;13:57.

22. Panwala CM, Jones JC, Viney JL. A novel model of inflammatory bowel disease: mice deficient for the multiple drug resistance gene, mdr1a, spontaneously develop colitis. JImmunol. 1998;161(10):5733-5744.

23. Wilk JN, Bilsborough J, Viney JL. The mdr1a mouse model of spontaneous colitis: a relevant and appropriate animal model to study inflammatory bowel disease. Immunol Res. 2005;31(2):151-159.

24. Paterson S, Cordero R, McCulloch S, Houldsworth P. Analysis of urine for drugs of abuse using mixed-mode solid-phase extraction and gas chromatography-mass spectrometry. Ann Clin Biochem. 2000;37(Pt 5):690-700.

25. Yusa K, Tsuruo T. Reversal mechanism of multidrug resistance by verapamil: direct binding of verapamil to P-glycoprotein on specific sites and transport of verapamil outward across the plasma membrane of K562/ADM cells. Cancer Res. 1989;49(18):5002-5006.

26. McGuinness D, et al. Characterizing cannabinoid CB2 receptor ligands using DiscoveRx PathHunter beta-arrestin assay. J Biomol Screen. 2009;14(1):49-58.

27. Ryberg E, et al. The orphan receptor GPR55 is a novel cannabinoid receptor. Br J Pharmacol. 2007;152(7):1092-1101.

28. Syed SK, et al. Regulation of GPR119 receptor activity with endocannabinoid-like lipids. Am J Physiol Endocrinol Metab. 2012;303(12):E1469-E1478.

29. DiPatrizio NV. Endocannabinoids in the gut. Cannabis Cannabinoid Res. 2016;1(1):67-77.

30. Turcotte C, Blanchet MR, Laviolette M, Flamand $\mathrm{N}$. The $\mathrm{CB} 2$ receptor and its role as a regulator of inflammation. Cell Mol Life Sci. 2016;73(23):4449-4470.

31. Patricelli MP, Cravatt BF. Characterization and manipulation of the acyl chain selectivity of fatty acid amide hydrolase. Biochemistry. 2001;40(20):6107-6115.

32. Dinh TP, et al. Brain monoglyceride lipase participating in endocannabinoid inactivation. Proc Natl Acad Sci U S A. 2002;99(16):10819-10824.

33. Kurihara R, et al. Effects of peripheral cannabinoid receptor ligands on motility and polarization in neutrophil-like HL60 cells and human neutrophils. J Biol Chem. 2006;281(18):12908-12918.

34. McHugh D, Tanner C, Mechoulam R, Pertwee RG, Ross RA. Inhibition of human neutrophil chemotaxis by endogenous cannabinoids and phytocannabinoids: evidence for a site distinct from CB1 and CB2. Mol Pharmacol. 2008;73(2):441-450.

35. Chicca A, Marazzi J, Nicolussi S, Gertsch J. Evidence for bidirectional endocannabinoid transport across cell membranes. J Biol Chem.
2012;287(41):34660-34682.

36. Zoratti C, Kipmen-Korgun D, Osibow K, Malli R, Graier WF. Anandamide initiates $\mathrm{Ca}(2+)$ signaling via $\mathrm{CB} 2$ receptor linked to phospholipase $\mathrm{C}$ in calf pulmonary endothelial cells. Br J Pharmacol. 2003;140(8):1351-1362.

37. Di Marzo V, De Petrocellis L. Why do cannabinoid receptors have more than one endogenous ligand? Philos Trans R Soc Lond, B, Biol Sci. 2012;367(1607):3216-3228.

38. Tanasescu R, Constantinescu CS. Cannabinoids and the immune system: an overview. Immunobiology. 2010;215(8):588-597.

39. Gonsiorek W, Lunn C, Fan X, Narula S, Lundell D, Hipkin RW. Endocannabinoid 2-arachidonyl glycerol is a full agonist through human type 2 cannabinoid receptor: antagonism by anandamide. Mol Pharmacol. 2000;57(5):1045-1050.

40. Console-Bram L, Marcu J, Abood ME. Cannabinoid receptors: nomenclature and pharmacological principles. Prog Neuropsychopharmacol Biol Psychiatry. 2012;38(1):4-15.

41. Staley EM, Schoeb TR, Lorenz RG. Differential susceptibility of P-glycoprotein deficient mice to colitis induction by environmental insults. Inflamm Bowel Dis. 2009;15(5):684-696.

42. Singh UP, Singh NP, Singh B, Price RL, Nagarkatti M, Nagarkatti PS. Cannabinoid receptor-2 (CB2) agonist ameliorates colitis in $\mathrm{IL}-10(-/)$ mice by attenuating the activation of $\mathrm{T}$ cells and promoting their apoptosis. Toxicol Appl Pharmacol. 2012;258(2):256-267.

43. Storr MA, Keenan CM, Zhang H, Patel KD, Makriyannis A, Sharkey KA. Activation of the cannabinoid 2 receptor (CB2) protects against experimental colitis. Inflamm Bowel Dis. 2009;15(11):1678-1685.

44. Izzo AA, Sharkey KA. Cannabinoids and the gut: new developments and emerging concepts. Pharmacol Ther. 2010;126(1):21-38.

45. Kunjachan S, Rychlik B, Storm G, Kiessling F, Lammers T. Multidrug resistance: Physiological principles and nanomedical solutions. Adv Drug Deliv Rev. 2013;65(13-14):1852-1865.

46. Nieri P, Romiti N, Adinolfi B, Chicca A, Massarelli I, Chieli E. Modulation of P-glycoprotein activity by cannabinoid molecules in HK-2 renal cells. Br J Pharmacol. 2006;148(5):682-687.

47. Miller AM, Stella N. CB2 receptor-mediated migration of immune cells: it can go either way. Br J Pharmacol. 2008;153(2):299-308.

48. Schicho R, Storr M. Cannabis finds its way into treatment of Crohn's disease. Pharmacology. 2014;93(1-2):1-3.

49. Bannenberg G, Serhan CN. Specialized proresolving lipid mediators in the inflammatory response: An update. Biochim Biophys Acta. 2010;1801(12):1260-1273.

50. Rossaint J, Nadler JL, Ley K, Zarbock A. Eliminating or blocking 12/15-lipoxygenase reduces neutrophil recruitment in mouse models of acute lung injury. Crit Care. 2012;16(5):R166.

51. Haile LA, et al. Myeloid-derived suppressor cells in inflammatory bowel disease: a new immunoregulatory pathway. Gastroenterology. 2008;135(3):871-881.

52. Jung $P$, et al. Isolation and in vitro expansion of human colonic stem cells. Nat Med. 
2011;17(10):1225-1227.

53. Sato T, et al. Single Lgr5 stem cells build cryptvillus structures in vitro without a mesenchymal niche. Nature. 2009;459(7244):262-265.

54. Miyoshi H, Stappenbeck TS. in vitro expansion and genetic modification of gastrointestinal stem cells in spheroid culture. Nat Protoc. 2013;8(12):2471-2482.

55. VanDussen KL, et al. Development of an enhanced human gastrointestinal epithelial culture system to facilitate patient-based assays. Gut. 2015;64(6):911-920.
56. Mócsai A, et al. G-protein-coupled receptor signaling in Syk-deficient neutrophils and Mast cells. Blood. 2013;101(10):4055-4163.

57. Hue S, et al. Interleukin-23 drives innate and $T$ cell-mediated intestinal inflammation. J Exp Med. 2006;203(11):2473-2483. 